\title{
Optical Filters Utilizing Ion Implanted Bragg Gratings in SOI Waveguides
}

\author{
M. P. Bulk, ${ }^{1}$ A. P. Knights, ${ }^{1}$ P. E. Jessop, ${ }^{1}$ P. Waugh, ${ }^{2}$ R. Loiacono, ${ }^{2}$ G. Z. Mashanovich, ${ }^{2}$ \\ G. T. Reed, ${ }^{2}$ and R. M. Gwilliam² \\ ${ }^{1}$ Department of Engineering Physics, McMaster University, 1280 Main Street West, Hamilton, ON, Canada L8S 4J1 \\ ${ }^{2}$ Advanced Technology Institute, University of Surrey, Guildford, Surrey GU2 4EL, UK
}

Correspondence should be addressed to A. P. Knights, aknight@mcmaster.ca

Received 6 January 2008; Accepted 13 March 2008

Recommended by Pavel Cheben

The refractive index modulation associated with the implantation of oxygen or silicon into waveguides formed in silicon-oninsulator (SOI) has been investigated to determine the feasibility of producing planar, implantation induced Bragg grating optical filters. A two-dimensional coupled mode theory-based simulation suggests that relatively short grating lengths, on the order of a thousand microns, can exhibit sufficient wavelength suppression, of $>10 \mathrm{~dB}$, using the implantation technique. Fabricated planar implanted slab-guided SOI waveguides demonstrated an extinction of $-10 \mathrm{~dB}$ for TE modes and $-6 \mathrm{~dB}$ for TM modes for the case of oxygen implantation. Extinctions of $-5 \mathrm{~dB}$ and $-2 \mathrm{~dB}$ have been demonstrated with silicon implantation.

Copyright (c) 2008 M. P. Bulk et al. This is an open access article distributed under the Creative Commons Attribution License, which permits unrestricted use, distribution, and reproduction in any medium, provided the original work is properly cited.

\section{INTRODUCTION}

The preliminary work of Soref et al. and the subsequent study by Pogossian et al. demonstrated the ability to produce single mode optical waveguides in silicon at telecommunication wavelengths with cross-sectional dimensions compatible with standard photolithography $[1,2]$. Immediate interest pursued as this structure was a suitable candidate for lowloss, high-index-contrast waveguides capable of routing optical signals at the wafer scale. The integration of optical components on a silicon platform also had the added benefits of low-cost large-scale manufacturability utilizing existing CMOS facilities and the possibility of amalgamating electrical and optical functionality on a single wafer.

Consequently, the miniaturized counterparts of many bulk optical components have been realized in silicon. These include detectors, couplers, filters, and sensors [3-6]. It is of particular interest to control the propagation of individual optical wave-fronts in such devices. One method to achieve wavelength dispersion exploits the unique behavior of optical waves propagating in a medium containing a periodically modulated refractive index. Indeed, this approach has found extensive use in such areas as photonic crystals, distributed feedback (DFB) lasers and optical spectroscopy. In onedimension, the perturbation is referred to as a grating and serves as a robust and flexible tool in photonics technology. Depending on the desired outcome, gratings can be implemented to acts as couplers, reflectors, mode converters, optical filters among others [7].

In the case of silicon-on-insulator (SOI) waveguides, a surface relief structure provides the most straightforward method for fabrication of an integrated Bragg grating. Such devices are inherently passive, although electrically tuneable gratings based on the plasma dispersion effect have been developed [8] along with those which are thermooptically tuned [9]. The Bragg grating itself is typically formed through a lithographic patterning technique and an etching sequence, although, relatively recently, focused ion beam milling has been used to realize higher-order mode conversion and filtering in SOI [10]. Perhaps the benchmark for performance of gratings in SOI waveguides remains the work of Murphy et al. [11]. There, surface relief gratings with a period of $223 \mathrm{~nm}$ were formed in $3 \mu \mathrm{m}$ high SOI rib waveguides via interference photolithography and plasma etching to a depth of $150 \mathrm{~nm}$. The authors demonstrated an optical transmission dip of $8 \mathrm{~dB}$ at the resonant wavelength 
of $1543 \mathrm{~nm}$, for the fundamental TE mode. The device had a transmission loss of nearly $10 \mathrm{~dB}$ which the authors attributed to sidewall scattering. The biggest drawback of the fabrication method leading to surface relief is the inherent deviation from a planar surface, limiting the ability of further processing. This is particularly problematic in nanophotonics, where the required thin processing layers are quickly skewed by alterations of wafer topography.

An alternative method which we propose here involves the well-established ion implantation process to introduce dopants and/or structural modifications to the crystalline lattice. By selectively introducing ions using a masking process, the refractive index can be periodically modified to produce a Bragg grating. The implantation process can alter the refractive index through either compound formation, as in the case of oxygen implantation, or chemically inert structural damage as achieved by self-irradiation [12, 13]. Apart from small volumetric changes, the result is a Bragg grating retaining a nearly planar surface. This makes it a very interesting candidate for photonic applications possessing surface sensitive topography, such as wafer-bonding techniques required for three-dimensional photonic circuits. Therefore, this paper investigates the formation of Bragg gratings via implantation of oxygen and silicon into SOI. To the authors' knowledge, this preliminary work is the first report of integrated Bragg gratings in SOI waveguides formed via ion implantation.

\section{ION IMPLANTED GRATING DESIGN AND CONSIDERATIONS}

Some authors have already pointed out the possibility of fabricating isolated buried oxide regions through a high dose oxygen implantation [14] in order to create hybrid $\mathrm{SOI} /$ silicon wafers for CMOS applications in a manner similar to the buried oxide formed in the separation by IMplantation of OXygen (SIMOX) process [12]. Such formation is generally conducted using doses around $10^{18}$ ions $/ \mathrm{cm}^{2}$, with energies of $150-200 \mathrm{keV}$ and at high temperatures $\left(65^{\circ} \mathrm{C}\right)$ in order to prevent amorphization [15]. Traditional SIMOX processes need high dose implants in order to obtain a stoichiometric oxide. In the case of grating fabrication, high dose is still a desirable process parameter, whereas the implant energy will be lower as the oxide layer needs not to be buried but close to the wafer surface. For this reason, implantation energy can be lowered to $20-30 \mathrm{keV}$. Following the implantation process, the substrate generally contains a series of extended defects. Depending on the implant condition these defects may include stacking faults, dislocation loops, amorphous clusters, oxygen precipitates, and columnar defects [16]. In general, SIMOX process requires postimplant annealing treatment up to $1300^{\circ} \mathrm{C}$. The postimplant anneal is essential in order to finalize oxide formation, as it stimulates oxygen diffusion and $\mathrm{SiO}_{2}$ formation via Ostwald Ripening [17], while repairing lattice damage. By this mechanism, small faulty zones and precipitates tend to coalesce into bigger aggregations in order to achieve a more favorable energy configuration. Although the structures reported here have not received such a high-

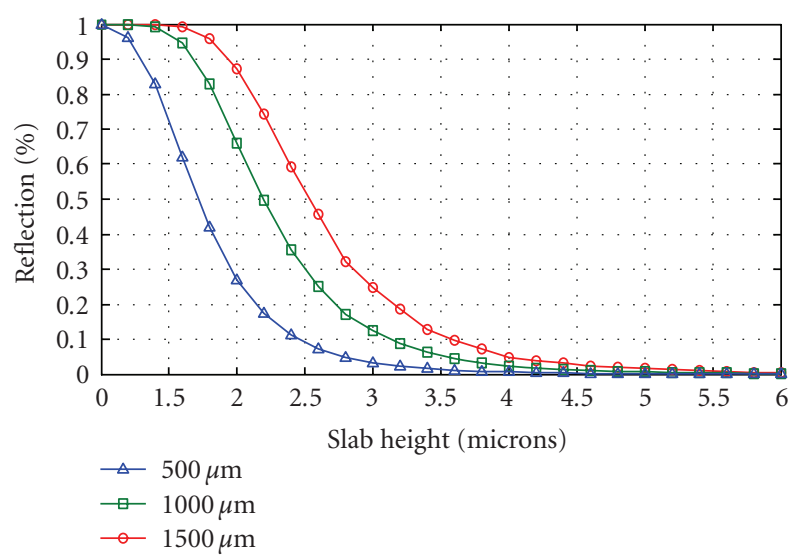

FIGURE 1: Coupled mode theory prediction of peak reflected power as a function of the silicon slab waveguide height for grating lengths of 500,1000 , and $1500 \mu \mathrm{m}$. Simulations are performed with regard to amorphous silicon induced via self-implantation. The depth of amorphous silicon is taken to be $100 \mathrm{~nm}$.

temperature anneal, it is likely that isolated precipitates of $\mathrm{SiO}_{2}$ are formed during the high temperature implantation stage.

In contrast, the low temperature self-irradiation of silicon results in the production and accumulation of point defects, defect complexes, and locally amorphous regions until it becomes thermodynamically favorable for the lattice to undergo a transition from the crystalline to amorphous state [18]. Of particular interest to optical devices is the ensuing increase of the index of refraction as a result of damage accumulation. The increase continues until the amorphous transition, at which point the refractive index saturates to a value approximately 0.27 higher (for a wavelength of around $1550 \mathrm{~nm}$ ) than that of crystalline silicon [19]. It should be noted that although propagation losses tend to be high, amorphous silicon $(a-\mathrm{Si})$ has demonstrated guiding capabilities. Therefore, if applied periodically, $a-$ Si serves as a unique candidate for grating structures.

In order to achieve a first-order Bragg grating in silicon, the Bragg condition,

$$
\Lambda=\frac{\lambda}{2 N_{\mathrm{eff}}}
$$

requires a period $(\Lambda)$ of $222 \mathrm{~nm}$, assuming a wavelength $(\lambda)$ of $1550 \mathrm{~nm}$ and an effective index $\left(N_{\text {eff }}\right)$ of approximately 3.48. This poses a fabrication challenge since the lateral damage/straggle must remain below $55 \mathrm{~nm}$ in order to achieve adequate modulation. Since the implantation profiles depend on the implantation energy, dose, temperature, implantation angle, and ion mass, the lateral profile can be manipulated by varying these parameters.

The implantation profiles can be construed using ion implantation simulators. In the case of the oxygen implantation, in order to achieve a uniform dopant implantation to a depth of $70 \mathrm{~nm}$ and retain adequate lateral modulation, it was determined that a dual implant process was required. The first implant utilized ion energy of $20 \mathrm{keV}$ with a dose 


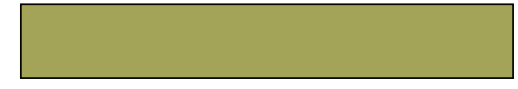

(i) Silicon-on-insulator wafer

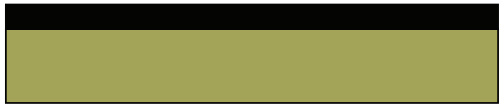

(ii) Silicon nitride (LPCVD at $\left.740^{\circ} \mathrm{C}\right)$

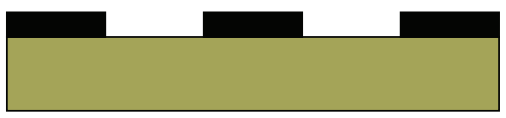

(iii) E-beam pattern $222 \mathrm{~nm}$ grating

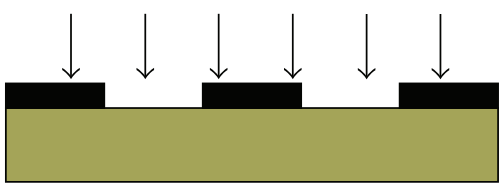

(iv) Oxygen implantation

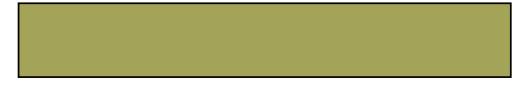

(i) Silicon-on-insulator wafer

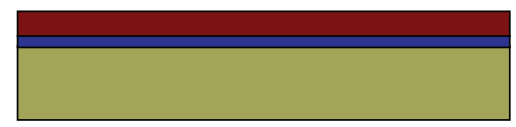

(ii) $30 \mathrm{~nm}$ thermal oxide and $100 \mathrm{~nm}$ photoresist

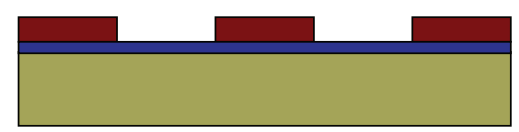

(iii) Holographically defined $220 \mathrm{~nm}$ grating

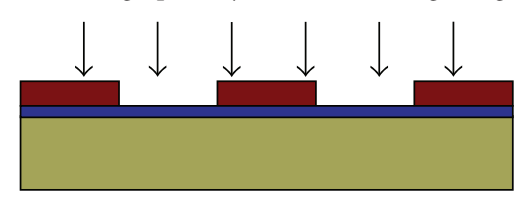

(iv) Silicon implantation

(a)

(b)

FIgURE 2: Fabrication process of planar SOI gratings for the case of (a) oxygen implantation and (b) silicon implantation.

of $1.6 \times 10^{17} \mathrm{ions} / \mathrm{cm}^{2}$. A subsequent surface implant was carried out at $10 \mathrm{keV}$ with a dose of $0.8 \times 10^{17} \mathrm{ions} / \mathrm{cm}^{2}$.

For silicon self-irradiation, and implanting at a temperature of $77 \mathrm{~K}$ to minimize dynamic annealing, a dose of approximately $10^{15}$ ions $/ \mathrm{cm}^{-2}$ is required to induce amorphization [18]. The total damage to the lattice is characterized by the number of displacements per unit volume of the crystal. When the defect contributions are higher than a critical value, the substrate can be approximated as amorphous. Although reported values vary, lattice displacement concentrations in the range of $10 \%$ to $25 \%$ of the silicon density appear to satisfy this condition [20,21].

Assuming an impenetrable masking layer, it was determined that to provide suitable lateral modulation the implant energy should remain below approximately $60 \mathrm{keV}$. The $60 \mathrm{keV}$ implant limit also determines the extent of the grating depth. Using similar analysis, a modulated index is expected to exist to a depth of approximately $150 \mathrm{~nm}$. Since the degree of coupling from the forwards to the backwards modes depends directly on the overlap integral of the mode with the grating region, it is important to quantify the suitability of the depth limitation.

A two-dimensional coupled mode theory (CMT) approach assuming TE modes in a slab SOI waveguide, with an upper-cladding of air, was applied to approximate the strength of the grating [22]. Assuming an implantation dose capable of rendering the silicon amorphous and taking the depth of the implant to be fixed at $100 \mathrm{~nm}$, the degree of modal overlap can be altered by varying the slab height. This is analogous to changing the silicon over-layer height. Figure 1 depicts the maximum reflected power for different grating lengths while varying the slab height, assuming a lossless medium for simplicity. From the figure, it is apparent that over $90 \%$ of the power is expected to be reflected at the resonant wavelength for slab heights on the order of $2 \mu \mathrm{m}$ and a grating length of approximately $1500 \mu \mathrm{m}$. Similar analysis was performed assuming a 70-nm deep grating consisting of a periodic oxide (as opposed to amorphous silicon) and grating lengths of $1000 \mu \mathrm{m}$ were found to result in approximately $35 \mathrm{~dB}$ reflections.

\section{FABRICATION}

The fabrication process is depicted schematically in Figure 2 for the cases of the oxygen implant and the silicon implant. For the oxygen implanted samples, a 100-nm layer of silicon nitride was deposited onto a $1.5 \mu \mathrm{m}$ over-layer SOI wafer using LPCVD at $740^{\circ} \mathrm{C}$. Electron-beam lithography then provided the necessary resolution to produce the $222 \mathrm{~nm}$ gratings in the silicon nitride layer to a length of $1000 \mu \mathrm{m}$. The patterned silicon nitride layer then served as the implantation mask. The samples were subsequently implanted following the dual implantation process formulated above at an elevated temperature such that amorphization was prevented. Following implantation, the nitride mask was removed.

While fabricating oxygen implanted samples, several processing issues have been noticed that can negatively influence the device performance. First and foremost, the necessity of high dose implantation associated with this is sputtering and target erosion problems. In the current case, the nitride hardmask used for pattern definition tends to sputter as the implantation advances. This means that pattern definition becomes more and more uncertain by the termination of the implantation. The sputtering yield is greatest on the mask sidewalls, as the angle between the ion beam and the surface is not zero. Atomic force microscopy (AFM) measurements on the implanted target also showed 


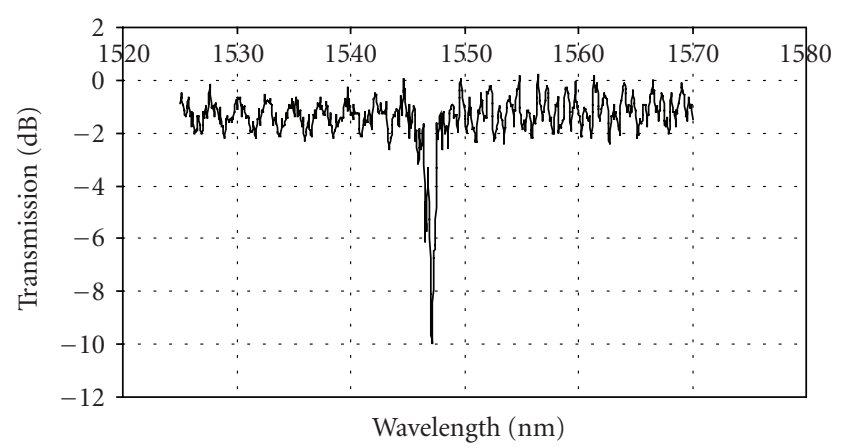

FIgURE 3: Experimental TE response of oxygen implant Bragg grating.

surface erosion of the order of $10 \mathrm{~nm}$. This means that the grating response could also be influenced by the surface modification.

The silicon implanted samples were implemented on a $2.2-\mu \mathrm{m}$ over-layer SOI wafer. The wafers were subject to a thermal oxidation resulting in a $40-\mathrm{nm}$ silicon dioxide layer. This layer serves as a buffer aiding the removal of the photoresist following implantation. A $7000-\mu \mathrm{m}$ grating pattern was produced holographically in photoresist using a Lloyd's mirror setup and a 325-nm HeCd laser. The photoresist layer then serves as the low-energy implantation mask. After exposure and development, scanning electron microscopy (SEM) images determined the photoresist thickness to be $100 \mathrm{~nm}$. Si implantation was carried out at energies of 50 and $70 \mathrm{keV}$ and a nominal dose of $1 \times 10^{15} \mathrm{ions} / \mathrm{cm}^{2}$ at $77 \mathrm{~K}$. Following implantation, the photoresist mask was removed, followed by the oxide buffer layer.

\section{RESULTS}

Optical transmission spectra were obtained utilizing a tunable laser operating in the range of 1525 to $1570 \mathrm{~nm}$. For the oxygen implanted samples, the signal was coupled to the waveguides using an objective lens. Light was then measured via a free-space power meter at the output. Prior to the objective lens, a beam splitter and half-wave plate were inserted to provide discrimination between TE and TM modes. The silicon implants were measured in transmission in a similar fashion, except for the use of a low-loss tapered optical fiber to butt couple light into the waveguide.

Figure 3 shows the TE mode of the "as-implanted" oxygen implanted sample, following the removal of the nitride mask. The peak response occurs at approximately $1547 \mathrm{~nm}$ for the TE polarized light and shows a discrimination of approximately $10 \mathrm{~dB}$. This appears to be a promising result, considering no post implantation annealing was preformed.

Using CMT analysis, the expected result on reflected power of a grating formed of $\mathrm{SiO}_{2}$ can be overlaid with the previous result to compare the expected and measured grating response. It is also possible to approximate the index of refraction responsible for the response in Figure 3 as a single fit factor, by assuming that the oxygen implant
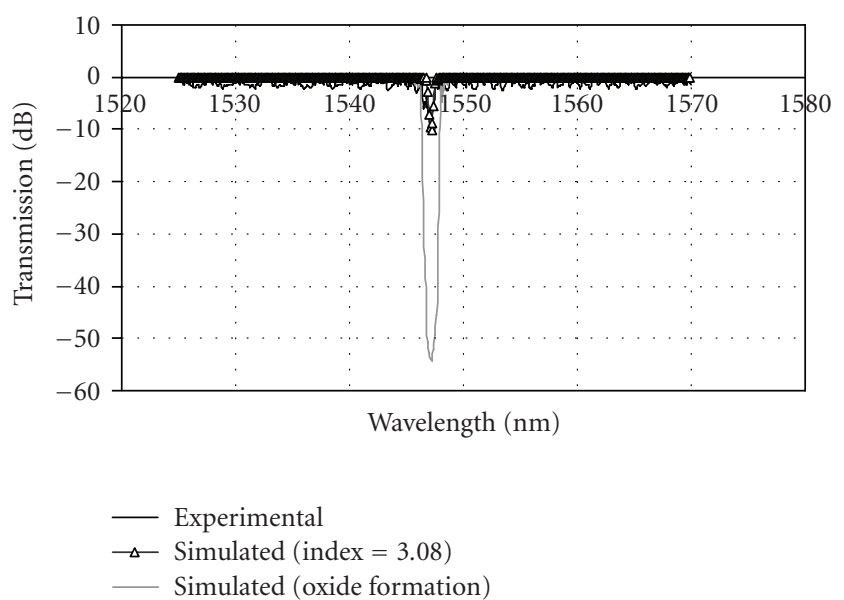

FIGURE 4: Simulated response of oxygen grating corresponding to experimental results.

achieves a depth of approximately $70 \mathrm{~nm}$. The result is shown in Figure 4.

From Figure 4, it is shown that the expected transmission drop, assuming complete oxide synthesis, is on the order of $-55 \mathrm{~dB}$, suggesting that the current results could be dramatically improved. Further, we approximate the index of refraction of the "as-implanted" region to lie at around 3.08. What is thus clear in this preliminary work is that the high dose, high temperature oxygen implantation, does not form continuous volumes of $\mathrm{SiO}_{2}$. In agreement with previous work [17], this should only be expected following postimplant annealing at around $1300^{\circ} \mathrm{C}$. An effort to fabricate gratings with fully formed oxide is currently in progress.

The resultant data for the $50 \mathrm{keV}$ and $70 \mathrm{keV}$ silicon implanted samples for TE light are shown in Figures 5 and 6 . The $50 \mathrm{keV}$ samples show a wavelength suppression of approximately $-5 \mathrm{~dB}$ at approximately $1530 \mathrm{~nm}$ with the oxide layer intact. Upon removing the oxide layer, the response drops to an extinction of around $-3 \mathrm{~dB}$. SEM images determined that no residual photoresist mask remained on the oxide and, therefore, suggested the damage inherent in the oxide layer contributes to the grating response. For the case of the $70 \mathrm{keV}$ implant with the oxide removed, the sample exhibited a $-5 \mathrm{~dB}$ suppression. The spectral response of the $50 \mathrm{keV}$ response is in agreement with the CMT model for the holographically defined $220 \mathrm{~nm}$ gratings, however, a $-3 \mathrm{~dB}$ suppression corresponds to an implanted index of only 3.493. This value is lower than expected for completely amorphized silicon and is either a result of ion straggle inflicting damage underneath the mask material reducing modulation or is a result of incomplete amorphization. For the $70 \mathrm{keV}$ samples, the implant depth is approximately $170 \mathrm{~nm}$, and the corresponding index to produce the $-5 \mathrm{~dB}$ extinction is 3.491 .

It is noted that for both the oxygen and silicon implanted grating devices there exists a deviation from the anticipated resonant wavelength of $1550 \mathrm{~nm}$. This can be attributed to 


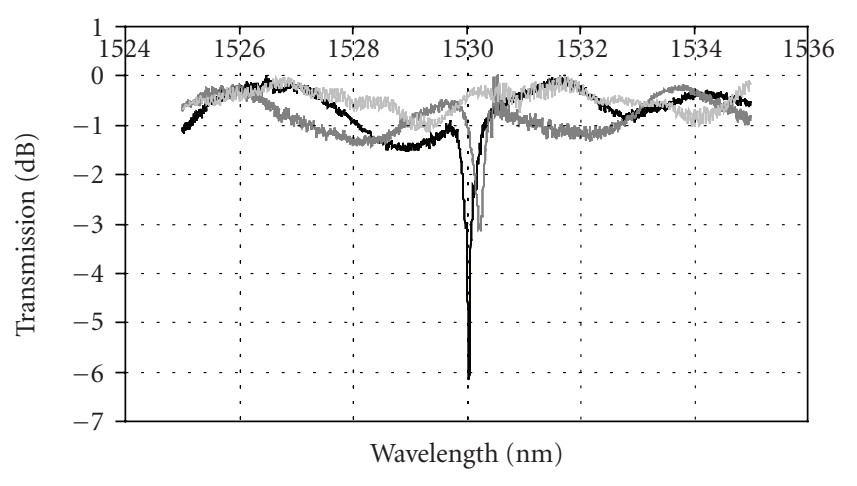

$-50 \mathrm{keV}$ implant with buffer oxidel
$-50 \mathrm{keV}$ implant oxide removed
$50 \mathrm{keV}$ implant $300^{\circ} \mathrm{C}$ anneal

FIgURE 5: Experimental TE response of $50 \mathrm{keV}$ silicon implanted Bragg grating.

an error associated with the lithographical fabrication. In the case of electron-beam lithography, we might anticipate a deviation in $\Lambda$ on the order of a few nm's, reflected in the small deviation from the resonance at $1550 \mathrm{~nm}$ of only $\sim 3 \mathrm{~nm}$, while in the holographic lithography process, a slight angular misalignment of the Lloyd's mirror setup of $1^{\circ}$ leads to a change in the Bragg wavelength of $\sim 25 \mathrm{~nm}$ (a direct result of the dependence of $\Lambda$ on the angle between the two mirrors). The measured resonance of $\sim 1530-1532 \mathrm{~nm}$ reflects a misalignment of the Lloyd's mirror setup of $\sim 1-2^{\circ}$. For this preliminary work, we find these results encouraging while recognizing that control of the lithographical process is critical to achieve precise resonance wavelengths.

In an attempt to improve the lateral modulation, a $300^{\circ} \mathrm{C}$ anneal for 10 minutes was performed on the silicon implanted samples. This annealing process was aimed to remove primary defects such as di-interstitials and divacancies (which can be found at the end of the implanted range in ion beam amorphized silicon) while retaining the amorphous structure itself [18]. As a result, amorphous pockets should remain and, in effect, be defined with a sharper amorphous/crystalline boundary. The results of the annealing on the transmission spectra are shown in Figures 5 and 6 . It is seen that the grating response is essentially quenched in both cases. This strongly suggests that the implantation conditions were not sufficient to invoke complete amorphization. Hence, the grating comprised of simple point defects in an otherwise crystalline structure. Therefore, the low-temperature anneal was enough to eliminate the point defects and lower the refractive index to near that of $\mathrm{c}$-Si and consequently eliminating the Bragg response. At the present time, further samples are being fabricated using a higher implantation dose, and a report of the annealing will be made in the future on the successful thermal processing of these grating structures.

No attention was paid to minimizing the optical propagation loss in these relatively crude slab waveguides. It is anticipated that Fresnel loss, mode mismatch between the

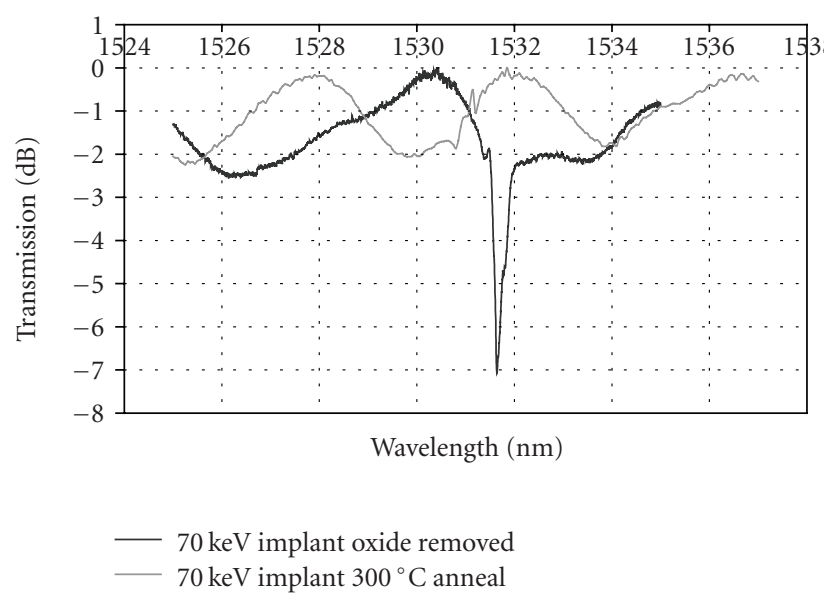

FIgURE 6: Experimental TE response of $70 \mathrm{keV}$ silicon implanted Bragg grating.

input beam and the waveguide, and incomplete collection of the output would introduce a significant "coupling" loss in this system of tens of $\mathrm{dB}$. Of some importance to these specific devices is the excess loss caused by the introduction of implantation induced lattice damage to the SOI (as in the case of the silicon implantation). This has been previously measured to be as much as $1000 \mathrm{dBcm}^{-1}$ for almost completely irradiated rib waveguide structures [23]. Given the shallow depth of the implantation used in this study (on the order of $100 \mathrm{~nm}$ ) and thus the relatively small overlap of the mode with the lattice damage, we expect an excess loss in the region of $10 \mathrm{~dB}$. Although this is undesirable, it is likely to be reduced significantly following low-temperature annealing [19]. The balance between minimizing optical loss and producing a strong interaction between the propagating mode and the grating will be dealt with in some detail in a future publication.

For this initial study, grating fabrication is limited to slab waveguides, although they will ultimately be of more practical importance when incorporated into rib waveguides. Whereas rib waveguides can be made effectively single mode by suitable choices of rib width and etch depth [2], slab waveguides of the thicknesses used in this study are highly multimode. The resonances shown in Figures 3 to 5 result from the coupling of light in the forward propagating fundamental mode into the reverse propagating fundamental mode. One might also expect to see resonances due to coupling from the forward propagating fundamental mode into higher-order reverse propagating modes or from higherorder forward propagating modes into reverse propagation of the same mode. The former type of higher-order resonance is observed in the case of relief gratings on rib waveguides where the higher-order modes are leaky [11]. We also expect to see this when implanted gratings are used in rib gratings. The higher-order resonances occur at wavelengths that are a few tens of nm lower than that of the fundamental resonance, corresponding to effective index differences of $1-2 \%$ between low-order modes in these 
structures. In this work, however, we observed neither type of higher-order resonance. We believe that the former type will be much weaker than in the rib waveguide case because of more complete mode orthogonality. The latter case is absent because the input light couples predominantly into the fundamental mode so that any individual higher-order mode has very little power in it to lose to resonant coupling.

\section{CONCLUSION}

This report demonstrated the viability of utilizing ion induced refractive index change in the manufacture of an optical filter in silicon waveguides. Response curves typical of passive grating filters were achieved, and extinction ratios of $-10 \mathrm{~dB}$ and $-7 \mathrm{~dB}$ were found for oxygen implants and $-5 \mathrm{~dB}$ and $-2 \mathrm{~dB}$ for silicon implants for TE and TM polarized light, respectively. From CMT analysis, it appears that the current results have the potential to be improved upon significantly. These implanted gratings could serve a useful role in future three-dimensional integrated photonics whose fabrication is contingent on surface sensitive bonding techniques.

\section{ACKNOWLEDGMENTS}

The authors would like to thank the Canadian Institute for Photonics Innovation (CIPI), the Natural Sciences and Engineering Research Council of Canada (NSERC), and the Ontario Centres of Excellence (OCE) for partially funding this work. We are also grateful to the members of the Centre for Emerging Device Technologies at McMaster University, and Interface Science Western, University of Western Ontario for assistance with device fabrication.

\section{REFERENCES}

[1] R. A. Soref, J. Schmidtchen, and K. Petermann, "Large singlemode rib waveguides in GeSi-Si and $\mathrm{Si}$-on-SiO $\mathrm{S}_{2}$," IEEE Journal of Quantum Electronics, vol. 27, no. 8, pp. 1971-1974, 1991.

[2] S. P. Pogossian, L. Vescan, and A. Vonsovici, "The single-mode condition for semiconductor rib waveguides with large cross section," Journal of Lightwave Technology, vol. 16, no. 10, pp. 1851-1853, 1998.

[3] J. D. B. Bradley, P. E. Jessop, and A. P. Knights, "Siliconwaveguide-integrated optical power monitor with enhanced sensitivity at $1550 \mathrm{~nm}$," Applied Physics Letters, vol. 86, no. 24, Article ID 241103, 3 pages, 2005.

[4] D. Taillaert, P. Bienstman, and R. Baets, "Compact efficient broadband grating coupler for silicon-on-insulator waveguides," Optics Letters, vol. 29, no. 23, pp. 2749-2751, 2004.

[5] P. D. Trinh, S. Yegnanarayanan, F. Coppinger, and B. Jalali, "Silicon-on-insulator (SOI) phased-array wavelength multi/demultiplexer with extremely low-polarization sensitivity," IEEE Photonics Technology Letters, vol. 9, no. 7, pp. 940942, 1997.

[6] G. N. Pearson and P. E. Jessop, "Silicon-on-insulator interferometric strain sensor," in Photonics Packaging and Integration III, vol. 4997 of Proceedings of SPIE, pp. 242-249, San Jose, Calif, USA, January 2003.

[7] H. Nishihara, M. Haruna, and T. Suhara, Optical Integrated Circuits, McGraw-Hill, New York, NY, USA, 1989.
[8] A. Cutolo, M. Iodice, A. Irace, P. Spirito, and L. Zeni, "An electrically controlled Bragg reflector integrated in a rib silicon on insulator waveguide," Applied Physics Letters, vol. 71, no. 2, pp. 199-201, 1997.

[9] S. Honda, Z. Wu, J. Matsui, et al., "Largely-tunable wideband Bragg gratings fabricated on SOI rib waveguides employed by deep-RIE," Electronics Letters, vol. 43, no. 11, pp. 630-631, 2007.

[10] D. J. Moss, V. G. Ta'eed, B. J. Eggleton, et al., "Bragg gratings in silicon-on-insulator waveguides by focused ion beam milling," Applied Physics Letters, vol. 85, no. 21, pp. 4860-4862, 2004.

[11] T. E. Murphy, J. T. Hastings, and H. I. Smith, "Fabrication and characterization of narrow-band Bragg-reflection filters in silicon-on-insulator ridge waveguides," Journal of Lightwave Technology, vol. 19, no. 12, pp. 1938-1942, 2001.

[12] G. T. Reed and A. P. Knights, Silicon Photonics: An Introduction, John Wiley \% Sons, Chichester, UK, 2004.

[13] J. E. Fredrickson, C. N. Waddell, W. G. Spitzer, and G. K. Hubler, "Effects of thermal annealing on the refractive index of amorphous silicon produced by ion implantation," Applied Physics Letters, vol. 40, no. 2, pp. 172-174, 1982.

[14] Y. Dong, M. Chen, J. Chen, X. Wang, and X. Wang, "Comparative study of SoI/Si hybrid substrates fabricated using highdose and low-dose oxygen implantation," Journal of Physics D, vol. 37, no. 13, pp. 1732-1735, 2004.

[15] K. Kajiyama, "Buried-oxide layer formation by high dose oxygen ion implantation into Si wafers: SIMOX (separation by implanted oxygen)," Applied Surface science, vol. 85, pp. 259264, 1995.

[16] A. G. Revesz and H. L. Hughes, "Properties of the buried oxide layer in SIMOX structures," Microelectronic Engineering, vol. 36, no. 1-4, pp. 343-350, 1997.

[17] Y. Li, C. D. Marsh, A. Nejim, R. J. Chater, J. A. Kilner, and P. L. F. Hemment, "SIMOX: processing, layer parameters design, and defect control," Nuclear Instruments and Methods in Physics Research B, vol. 99, no. 1-4, pp. 479-483, 1995.

[18] L. Pelaz, L. A. Marqués, and J. Barbolla, "Ion-beam-induced amorphization and recrystallization in silicon," Journal of Applied Physics, vol. 96, no. 11, pp. 5947-5976, 2004.

[19] M. J. A. de Dood, A. Polman, T. Zijlstra, and E. W. J. M. van der Drift, "Amorphous silicon waveguides for microphotonics," Journal of Applied Physics, vol. 92, no. 2, pp. 649-653, 2002.

[20] L. A. Christel, J. F. Gibbons, and T. W. Sigmon, "Displacement criterion for amorphization of silicon during ion implantation," Journal of Applied Physics, vol. 52, no. 12, pp. 7143-7146, 1981.

[21] W. P. Maszara and G. A. Rozgonyi, "Kinetics of damage production in silicon during self-implantation," Journal of Applied Physics, vol. 60, no. 7, pp. 2310-2315, 1986.

[22] A. Yariv, Introduction to Optical Electronics, Holt, Rinehart and Winston, New York, NY, USA, 1976.

[23] A. P. Knights and G. F. Hopper, "Effect of ion implantation induced defects on optical attenuation in silicon waveguides," Electronics Letters, vol. 39, no. 23, pp. 1648-1649, 2003. 

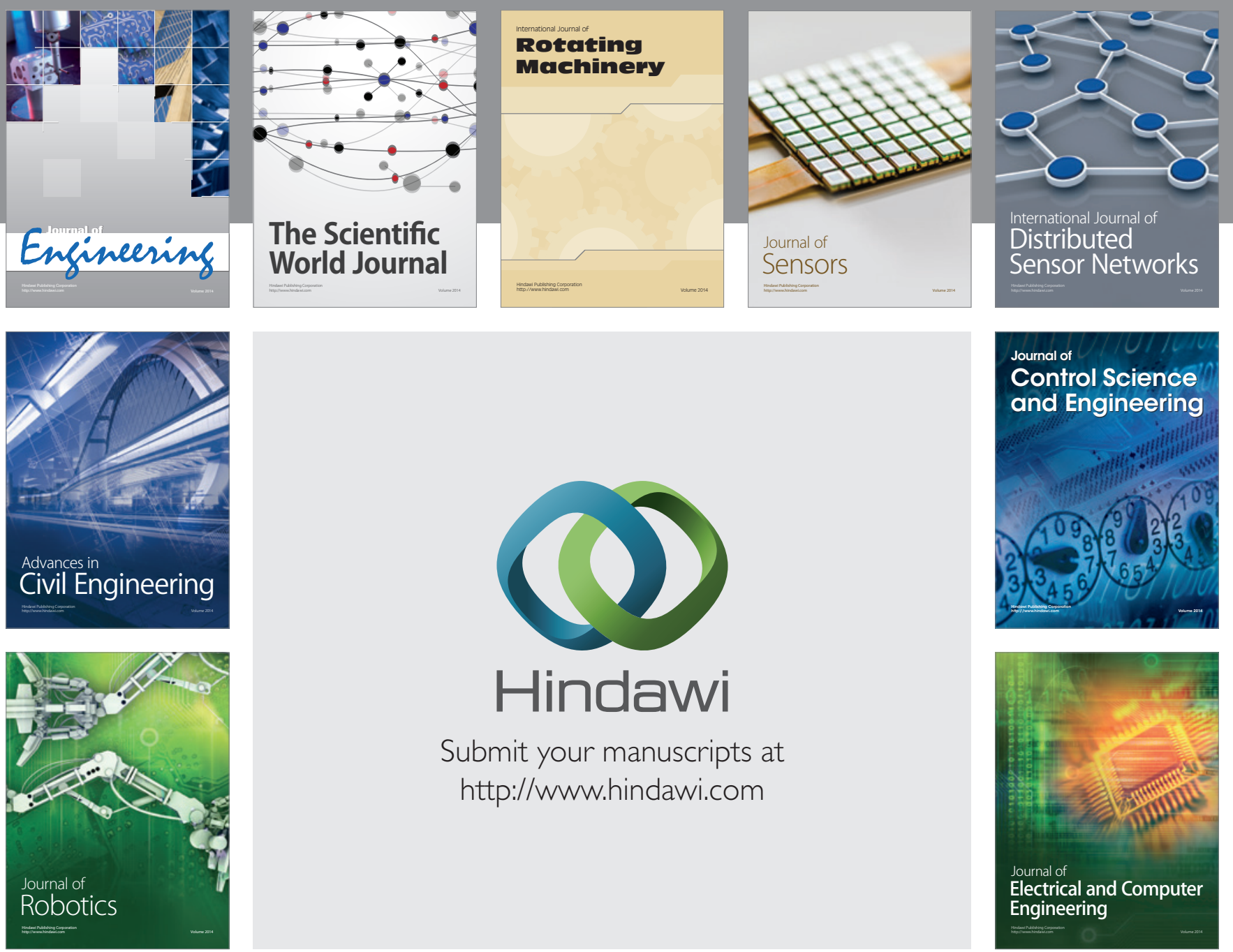

Submit your manuscripts at

http://www.hindawi.com
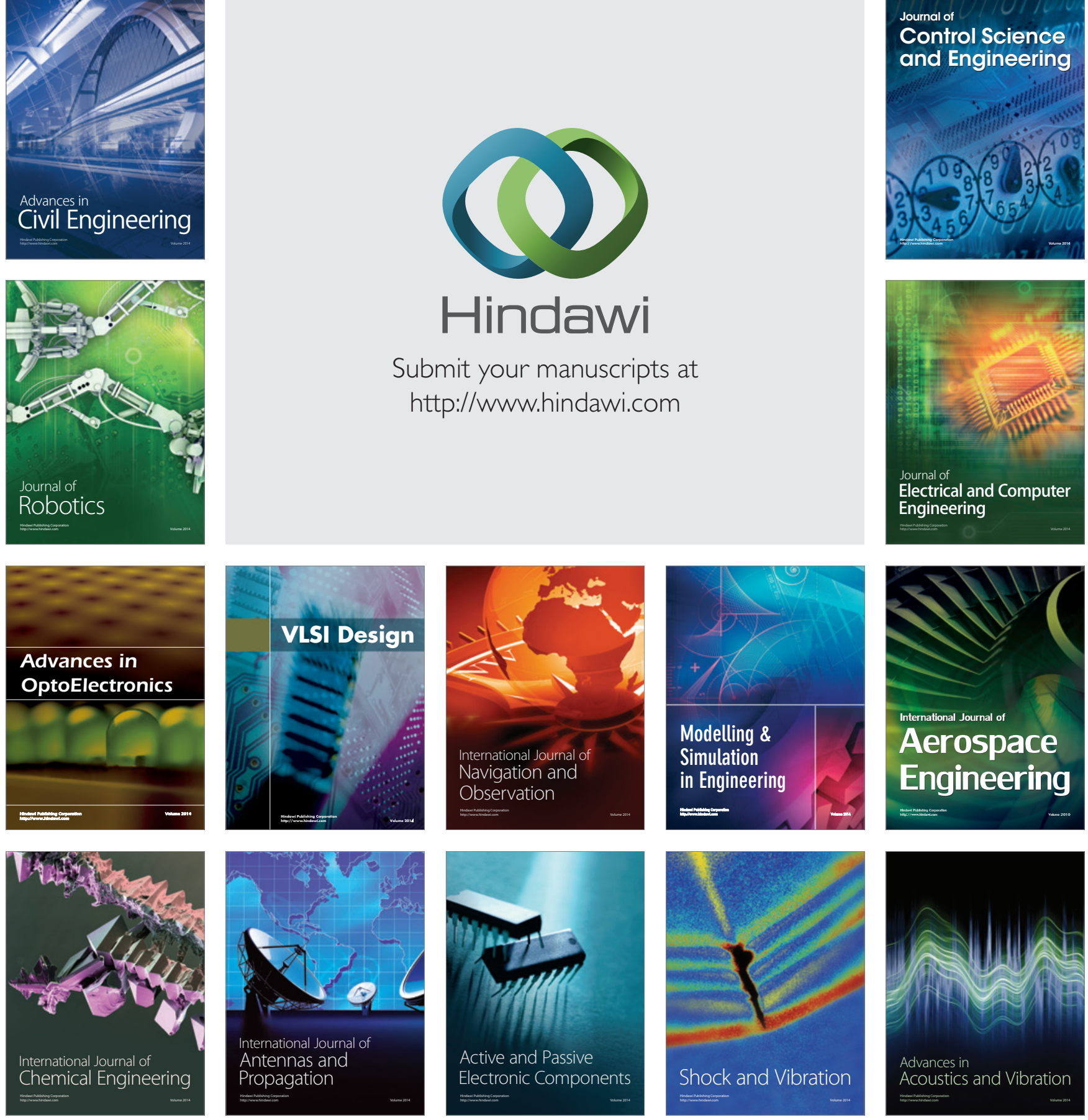